\title{
Passive Screening and Diagnosis of Sleeping Sickness with New Tools in Primary Health Services: An Operational Research
}

\author{
Philippe Mulenga · Pascal Lutumba · Yves Coppieters · \\ Alain Mpanya · Eric Mwamba-Miaka · Oscar Luboya · Faustin Chenge
}

Received: May 15, 2019 / Published online: July 15, 2019

(C) The Author(s) 2019

\section{ABSTRACT}

Introduction: The integration of human African trypanosomiasis (HAT) activities into primary health services is gaining importance as a result of the decreasing incidence of HAT and

Enhanced Digital Features To view enhanced digital features for this article go to https://doi.org/10.6084/ m9.figshare.8427905.

P. Mulenga $(\varangle) \cdot$ O. Luboya $\cdot$ F. Chenge

Faculty of Medicine \& School of Public Health, University of Lubumbashi, Lubumbashi, Democratic Republic of the Congo e-mail: drphilippe.mulenga@gmail.com; Philippe.Mulenga.Cilundika@ulb.ac.be

\section{P. Mulenga}

Department of Public Health, Institute of Tropical Medicine, Antwerp, Belgium

P. Mulenga $\cdot$ Y. Coppieters

School of Public Health, Université Libre de Bruxelles, Brussels, Belgium

\section{P. Lutumba}

Department of Tropical Medicine, University of Kinshasa, Faculty of Medicine, Kinshasa, Democratic Republic of the Congo

A. Mpanya · E. Mwamba-Miaka Programme National de Lutte contre la Trypanosomiase Humaine Africaine, Kinshasa, Democratic Republic of the Congo

F. Chenge

Centre de Connaissances en Santé en République Démocratique du Congo, Kinshasa, Democratic Republic of the Congo the ongoing developments of new screening and diagnostic tools. In the Democratic Republic of Congo, this integration process faces multiple challenges. We initiated an operational research project to document drivers and bottlenecks of the process.

Methods: Three health districts piloted the integration of HAT screening and diagnosis into primary health services. We analysed the outcome indicators of this intervention and conducted in-depth interviews with health care providers, seropositives, community health workers and HD management team members. Our thematic interview guide focused on factors facilitating and impeding the integration of HAT screening.

Results: The study showed a HAT-RDT-positive rate of $2.2 \%$ in Yasa Bonga, 2.9\% in Kongolo and $3 \%$ in Bibanga, while the proportion of reported seropositives that received confirmatory examinations was $76 \%, 45.6 \%$ and $68 \%$, respectively. Qualitative analyses indicated that some seropositives were unable to access the confirmation facility. The main reasons that were given included distance, RDT rupture, lack of basic screening equipment and financial barriers (additional hospital fees not included in free treatment course), fear of lumbar puncture and the perception of HAT as a disease of supernatural origin.

Conclusion: Passive screening using HAT RDTs in primary health services inevitably has some limitations. However, regarding the 
epidemiological context and some obstacles to integrated implementation, this cannot on its own be a relevant alternative to the elimination of HAT by 2020 .

Funding: We acknowledge the agency that provided financial support for this study, the Belgian Development Cooperation. The funder had no role in the study design, data collection and analysis, decision to publish or preparation of the manuscript. Philippe Mulenga received financial support thanks to a doctoral grant from the Belgian Development Cooperation under the FA4 agreement. Funding for the study and Rapid Service Fees was provided by the Epidemiology and Tropical Diseases Unit of the Institute of Tropical Medicine, Antwerp.

Keywords: Diagnosis; Human African trypanosomiasis; Integration; Operational research; Passive screening; Primary health services; Sleeping sickness

\section{INTRODUCTION}

Human African trypanosomiasis (HAT), or sleeping sickness, is a neglected, vector-borne tropical disease that affects 70 million people in sub-Saharan Africa [1, 2]. It is a parasite transmitted to humans through the bite of an infected tsetse fly. Two species of parasites can cause HAT: Trypanosoma brucei (T.b.) gambiense and T.b. rhodesiense. The most common form, caused by T.b. gambiense, accounts for $>97 \%$ of all HAT cases [3, 4]. In 2016, < 3000 T.b. gambiense cases were reported worldwide and $>80 \%$ of these are reported in the Democratic Republic of the Congo (DRC). Between 2000 and 2016, the annual number of cases drastically decreased from around 26,000 to $<3000$ [5]. This steady decline prompted the World Health Organisation (WHO) to include elimination of HAT T.b. gambiense as a public health problem by 2020 [6] in their plan. In 2012, this goal was included and endorsed by the London Declaration, which ensured continuation and expansion of programmes for the supply of drugs and other necessary interventions $[7,8]$.

The disease has two clinical stages: an initial or haemo-lymphatic stage and a second meningo-encephalitic stage. During the first stage, non-specific symptoms arise that are similar to malaria symptoms, a pathology that is often endemic in sub-Saharan Africa [9]. Patients who consult have usually reached the second, meningo-encephalitic stage characterised by neurological and psychiatric signs [10-12]. If undiagnosed and untreated, HAT is often fatal $[13,14]$.

Since HAT does not present any specific signs in the haemo-lymphatic phase, the first step in the diagnostic procedure is a serological test to detect trypanosome-specific antibodies. Serologically positive subjects are considered "seropositive" and subjected to parasitological confirmation tests [15]. The card agglutination test for trypanosomiasis (CATT) was the only serological test used in the field [5] until the rapid diagnostic test for HAT (HAT RDT) was developed [16]. The major advantages of the RDT are its affordability, being individually packaged for single use, not requiring external energy sources and being storable for 2 years at temperatures up to $40{ }^{\circ} \mathrm{C}[9,17]$, making it suitable for use in low-resource countries. However, HAT RDT positivity should always be confirmed by microscopy because of its low specificity (around 90\%) [17, 18]. The specificity of several microscopy techniques used for the confirmation of HAT is assumed to be $100 \%$ [19].

Control of T.b. gambiense HAT is based on two strategies: (1) case detection followed by treatment of the confirmed cases and (2) vector control [20]. Detection consists of active or passive case finding [21]. Active screening (AS) is carried out by mobile units (MUs) that move from village to village to examine whole village populations in HAT-endemic areas. Passive screening (PS) is performed in fixed health facilities for patients presenting with possible symptoms of HAT [22]. These facilities can be multi-purpose health care centres or specialised centres run by the programme [17]. The literature shows that control and surveillance of HAT should be based on the combined use of active and passive screening. This combination should depend on the epidemiological situation and the characteristics of the health system $[3,20,23]$. 
In the DRC, the integration of control and surveillance of HAT into the health system has been a national policy goal supported by the "National Strategy for Strengthening the Health System" for many years [24]. This process has been established progressively in most endemic provinces [25], but has faced some difficulties. Mitashi et al.'s survey in two health districts in Bandundu, DRC, showed that in rural areas where HAT is most endemic, well-equipped health centres with appropriate screening and diagnostic tools are rare [21]. The current development of new tools improves the potential for integration of control activities into primary health services [9]. A recent study in Uganda concluded that in fixed health centres patients with a negative malaria test result or who have not been cured after anti-malarial treatment are considered seropositives and are tested with the HAT RDT [9]. To improve its control strategies, in 2015, the National HAT Control Programme of the DRC (PNLTHA) made the integration of screening and diagnosis of HAT in primary health centres of endemic health districts one of its operational research priorities [26].

This study was thus initiated within this context. The main objective was to investigate the added value of passive HAT screening by using new diagnostic tools in primary health services. This exploration consisted of documenting the drivers and bottlenecks in the integration process. The efficiency of the integration process could contribute to improving the screening and diagnosis of HAT. As the elimination of HAT as a public health problem deadline approaches, it is also useful to investigate new strategies to ensure and above all maintain this objective.

\section{METHODS}

\section{Study Sites}

This operational research project was conducted from August 2016 to December 2018 in three health districts (HDs) where HAT is endemic. The HDs of Yasa Bonga, Bibanga and Kongolo are respectively situated in the DRC provinces

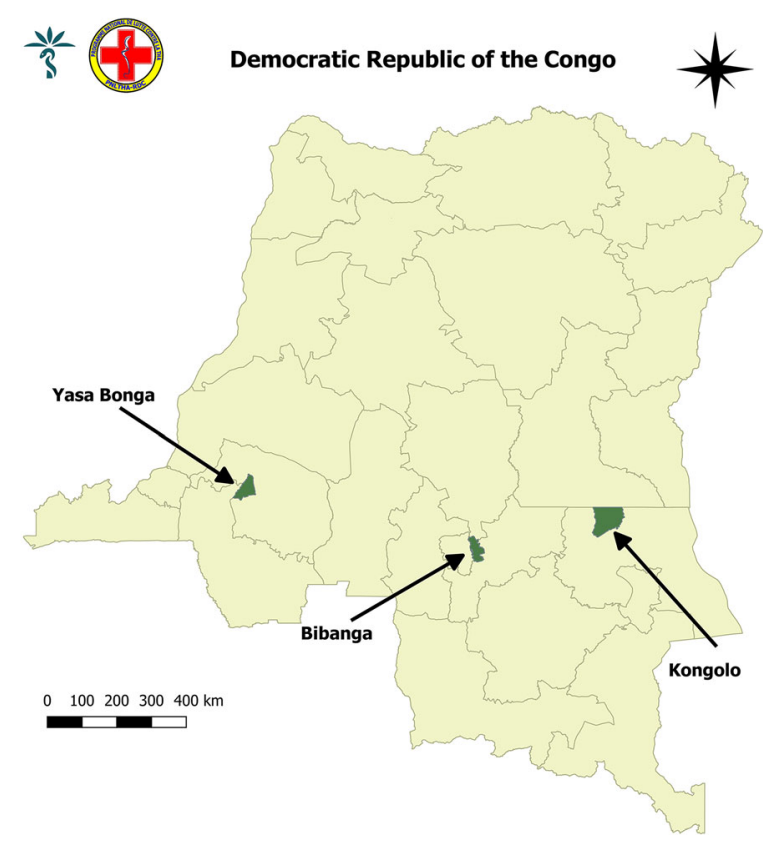

Fig. 1 Map of DRC with study sites: health districts Yasa Bonga, Kongolo and Bibanga (created using Quantum GIS 2.8 Las Palmas http://www.qgis.org)

of Kwilu, Kasaï Oriental and Tanganyika (Fig. 1). They were selected according to two key factors: (1) the number of reported HAT cases in 2015 (63 for Yasa Bonga, 33 for Bibanga, and 16 for Kongolo) and (2) their level of functionality (Kongolo and Bibanga have less well-equipped hospitals because they operate without external support, while hospitals in Yasa Bonga receive external support). These two key factors may influence the process of integrating screening and diagnosis of HAT into primary health services. The health services in these HDs are organised according to a pyramidal district system containing one general referral hospital (GRH) and primary health centres in the outskirts of the district. To provide health services closer to the population, some HDs installed secondary hospitals $(\mathrm{SH})$ where services similar to the GRH can be provided. In this survey, all public fixed health centres of the three HDs were included (Table 1). All public health centres and hospitals are supervised by the Central Health District Office. To set up the integration of HAT in the Democratic Republic of the Congo, the PNLTHA is implementing specialised sanitary structures in referral 
Table 1 Organisation of health services in the different health districts

\begin{tabular}{llll}
\hline Services & $\begin{array}{l}\text { HD Yasa } \\
\text { Bonga }\end{array}$ & $\begin{array}{l}\text { HD } \\
\text { Bibanga }\end{array}$ & $\begin{array}{l}\text { HD } \\
\text { Kongolo }\end{array}$ \\
\hline $\begin{array}{l}\text { Health centres (HC) } \\
\begin{array}{l}\text { Secondary hospitals } \\
(\mathrm{SH})\end{array}\end{array}$ & 3 & 16 & 24 \\
$\begin{array}{l}\text { General referral } \\
\text { hospital (GRH) }\end{array}$ & 1 & 1 & 1 \\
\hline
\end{tabular}

institutions in HDs. In the three HDs in the present survey, all specialised structures of the PNLTHA were added to the referral structures (GRH or SH).

The PNLTHA includes administrative (central and provincial management coordinations) and operational structures [specialised services of the PNLTHA such as mobile units, control, diagnostic and treatment centres (CDTC) and the primary health centres of the HDs that have integrated HAT control activities]. The organisation of control activities is entirely managed by the PNLTHAs. Reports from all health centres and specialised services are collected by the heads of the MUs. HDs can consult these reports, and the programme decides whether to use them for decision-making.

\section{Screening and Diagnostic Procedure Used}

We proceeded as follows for the introduction of HAT activities in the HDs: first, we organised a meeting of all stakeholders involved in each health district (PNLTHA, management team, partners, territorial administrators, actors in the health sector, community workers' representatives, etc.). The purpose of this meeting was to inform them about the introduction of HAT activities into the HDs. We subsequently set up technical training sessions targeting three groups according to predefined learning objectives: (1) a group consisting of HD management teams (training in clinical diagnosis, treatment, PS algorithm, RDT use and managerial aspects of HAT activities); (2) a second group comprising all health care providers (training in clinical diagnosis, treatment, PS algorithm, RDT use and usefulness of monthly reporting); (3) a last group consisting of the laboratory technicians of the referral structures (training in diagnostic confirmation techniques). The community health workers were briefed by the head nurse on site in their respective centres. During this survey, we used the HAT RDT SD Bioline, provided by our collaborating partners.

After training, the primary health centres received HAT RDTs while the referral facilities (GRH and SH) were provided with equipment to perform confirmation in addition to the RDTs. The central health district office managed the supply of tests and drugs. The research team closely collaborated with and monitored all activities with the HD management team and with PNLTHA.

Screening was initiated when a patient visited a primary health centre presenting with one or more signs or symptoms suggestive of HAT (fever, headache, pruritus, weakness, asthenia, anaemia and, to a lesser extent, hepato-splenomegaly) [3]. Since some of these signs or symptoms are also suggestive of malaria, patients were first subjected to a malaria RDT. If this was positive but the patient did not respond to treatment after 3 days, the patient received an HAT RDT. All patients presenting with newly emerging neuro-psychiatric symptoms and those with negative malaria RDT were given a HAT RDT. Patients who were found positive were considered "seropositive" and referred to a care facility equipped for parasitological confirmation. Follow-up of seropositives was done by transmitting the names of nonconfirmed seropositives to the PNLTHA on a monthly basis. This strategy allowed the PNLTHA to perform active retrieval of the patient for parasitological confirmation. If positive for parasitology, they received stage-specific treatment following the guidelines of the DRC-HAT treatment policy (Fig. 2). All the patients subjected to HAT RDT screening and whose results were negative were further examined depending on the health centre's technical set-up.

Confirmation centres used another algorithm to facilitate the laboratory technicians' tasks (Fig. 3) consisting of an examination of the seropositives (RDT positive). If lymph nodes 
sign or symptoms suggestive for HAT (fever, headache, pruritus, weakness, asthenia, anaemia and, to a lesser extent, hepato-splenomegaly)
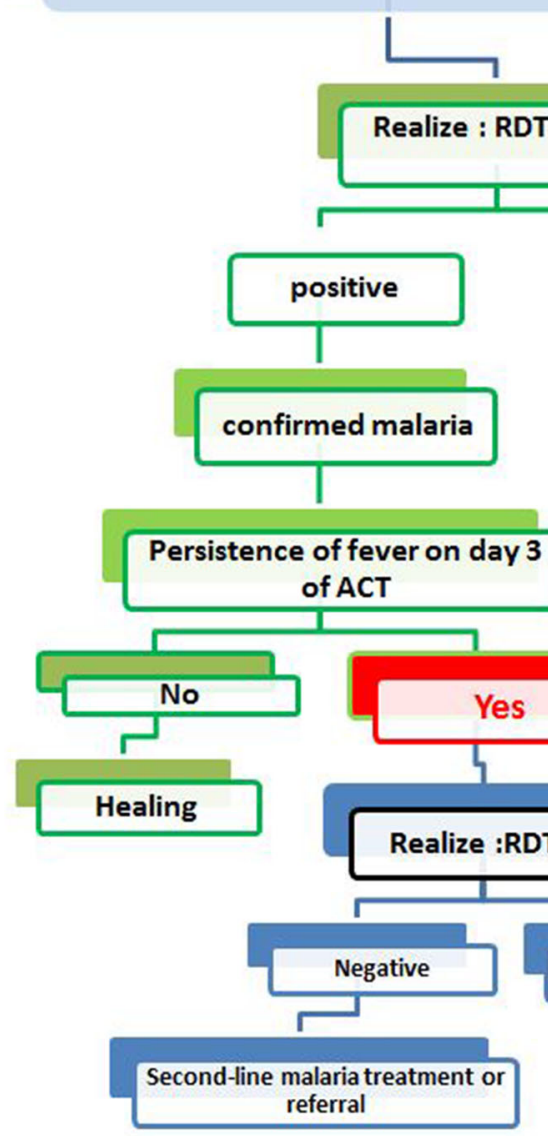

Fig. 2 Screening algorithm for human African trypanosomiasis in a primary health service. GP gland puncture, mAECT mini-Anion Exchange Centrifugation Technique,

were present in the back of the neck, the patient had to undergo a puncture of the lymph node to search for living parasites in the ganglion fluid. If this was negative or no lymph nodes were present, the laboratory technician drew venous blood and performed the mini-Anion Exchange Centrifugation Technique (mAECT). In HAT-confirmed subjects (positive lymph node aspirator, mAECT), the health care provider carried out a stage diagnosis (lumbar puncture), which determined the treatment course. Since reporting a HAT case is the responsibility
$R D T$ rapid diagnostic test, $T D$ tick drop, $A C T$ artemisinin-based combination therapy ()

of the programme, staff had to complete a new case report form and the PNLTHA patient form. If the mAECT was negative, the patient was referred to the hospital.

All first-stage patients were treated at the health centre. Second-stage patients received treatment in a referral facility.

The health district management team was responsible for formative supervision of the care providers and the planning of activities through their operational action plan. The HD benefited from technical support from PNLTHA experts 


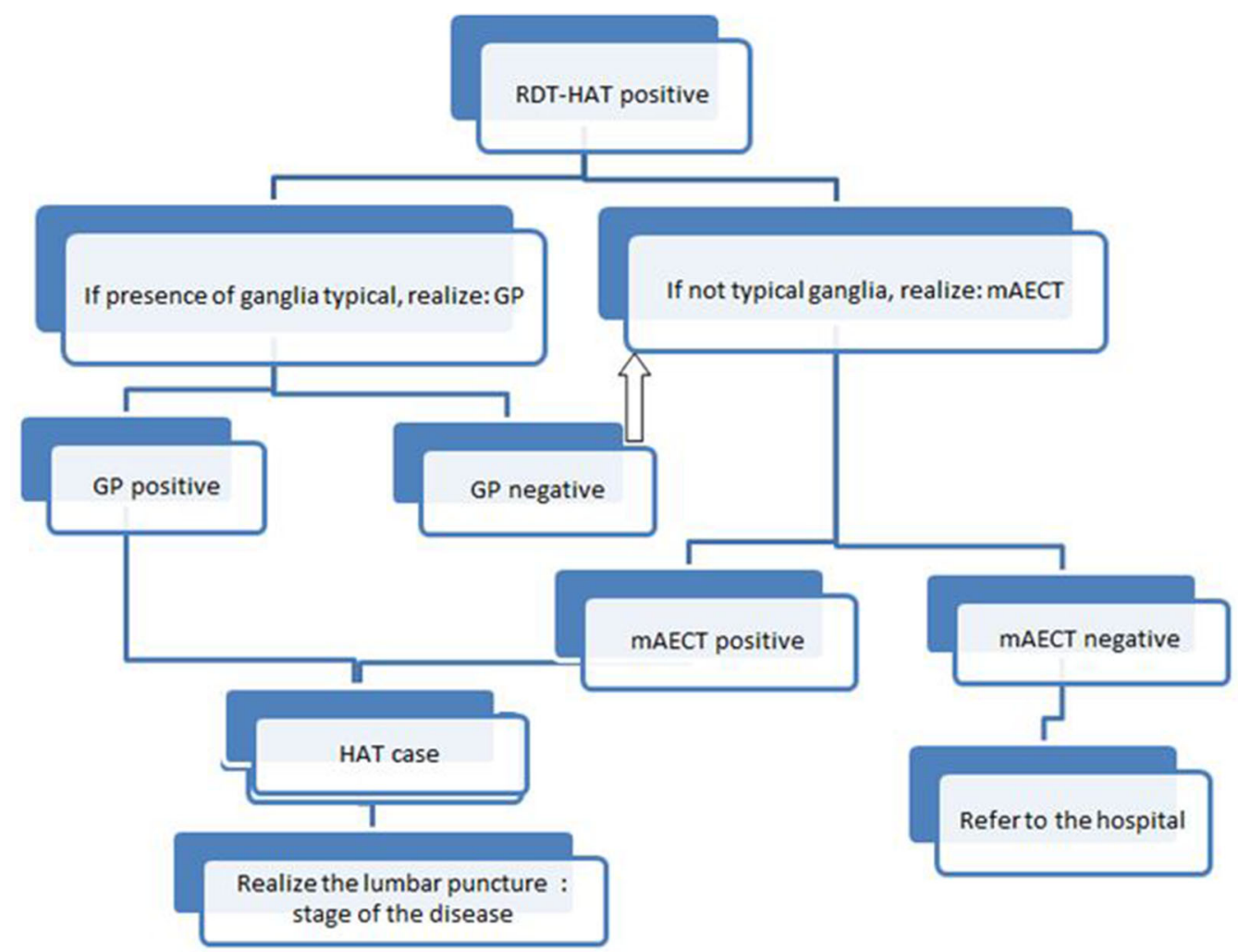

Fig. 3 Algorithm for HAT diagnosis in a confirmation structure. GP gland puncture, $m A E C T$ mini-Anion Exchange Centrifugation Technique

for supervision and monthly monitoring of their activities. As is the case for the other activities, the HC reported to the HD's central office.

\section{Data Collection and Analysis}

The following indicators were used to monitor screening and diagnostic activities in HDs: (1) health centre utilisation rate to give a rough idea of the functionality of the centre. The numerator of this rate is the number of new cases per year and the denominator is the size of the population in the HD; (2) the number of HAT RDTs performed in the centres to obtain the exact number of people who actually benefited from a HAT RDT; (3) the number of positive tests to estimate the number of seropositives; (4) the number of seropositives that reached the confirmation facility and received confirmatory examinations. This provided information on seropositive patients who had actually undergone parasitological examinations; (5) proportion of seropositives confirmed is the ratio between the number of seropositives confirmed parasitologically and the number of seropositives; (6) the screening rate: information on the relative additional workload due to the integration of HAT screening in primary health centres; (7) the HAT RDT-positive rate is the ratio of the number of positive HAT RDTs and the number of completed RDTs; (8) the HAT RDT availability rate: this indicator provides information about the time interval in which the test was available in each HD. This rate of the availability per product (RAP) is the percentage of the period during which the product is available for a considered time period; it is calculated considering the number of days of the stock shortage. It is calculated with the following expression: 
$\mathrm{RAP}=\frac{\text { number of days for the considered period }- \text { number of days of stock shortage }}{\text { number of days for the considered period }} \times 100$

All data were collected and analysed by the research team. We used Microsoft Excel 2013 software for recording and analysis and Stata/IC 14.1 software to analyse data on distances travelled by patients suspected of having HAT between screening and confirmation facilities. We used Student's $t$ test to compare the differences in the mean distances between the screening centre and confirmation centre. Levene's test was done before this to check the equivalence of the variances in the sample; a threshold of $5 \%$ was used and $p<0.05$ was considered significant.

To further document the factors that may positively or negatively influence the integration of HAT activities, qualitative data (in-depth interviews) were collected from those involved and/or in touch with HAT activities within the selected HDs (Table 2). The persons invited for interviews included health professionals, community health workers and seropositives. To know more about the reasoning behind attending the referral centre or not, we particularly invited seropositive patients who did not reach the confirmation facility. The data were collected between October and December 2018

Table 2 Interviewees on integrated passive screening in different health districts

\begin{tabular}{lllll}
\hline Category & $\begin{array}{l}\text { HD } \\
\text { Yasa } \\
\text { Bonga }\end{array}$ & $\begin{array}{l}\text { HD } \\
\text { Bibanga }\end{array}$ & $\begin{array}{l}\text { HD } \\
\text { Kongolo }\end{array}$ & Total \\
\hline $\begin{array}{l}\text { Doctors } \\
\text { Head nurses }\end{array}$ & 1 & 1 & 1 & 3 \\
$\begin{array}{l}\text { Supervisor nurses } \\
\text { of health }\end{array}$ & 1 & 1 & 2 & 6 \\
$\quad \begin{array}{l}\text { district } \\
\begin{array}{l}\text { Community } \\
\text { health workers }\end{array}\end{array}$ & 2 & 2 & 2 & 3 \\
\begin{tabular}{l} 
Seropositives \\
\hline
\end{tabular} & 4 & 3 & 3 & 6 \\
\hline
\end{tabular}

by the principal investigator (PI) and two observers who were trained in qualitative research methods. Participants were contacted well in advance to identify a suitable time for the interview. The research team developed thematic interview guides. To ensure the rigour and quality of the transcription process, qualified field staff transcribed and translated the material under the supervision of the PI. All local language interviews were translated into French. Quotations selected for this article were translated into English by the PI.

Interview data were analysed according to a thematic approach [27]. Consequently, the authors used an inductive approach towards the data transcripts, marking the text elements that provided answers to the research question (called open codes). These open codes were subsequently grouped into themes encompassing more general ideas from the interviews. These themes were again regrouped and used to analyse all the interviews. This provided an overview of the overall outcome of the qualitative part of this study. A conceptual diagram illustrated the relationships between the different themes. The NVivo 11 QSR software was used as an auxiliary tool to facilitate the analysis. The results were reported according to the consolidated criteria for qualitative research publications (COREQ) [28]. The quotes resulting from these interviews were identified by the interview code (INT), followed by a location code and a number, followed by the name of the function or the word "seropositive" for patients.

The study was approved by the Institutional Review Board Institute of Tropical Medicine Antwerp (no. 1156) and the Medical Ethics Committee of the University of LubumbashiDRC (UNILU/CEM/2017/004). We received authorisation from the Ministry of Public Health-DRC by the General Secretariat to carry out this study (MS no. 1251/SG/THA/2416/MK/ 
2017) and local leaders also gave their oral consent. The ethics committee approved the verbal consent procedure, documented by audio recording. The information collected from the participants was treated confidentially and anonymised.

\section{RESULTS}

First, we present an overview of patient followup over the 24-month period in which integration of HAT activities was implemented. We will subsequently present the results of the qualitative survey.

\section{Follow-Up of Screened and Diagnosed Patients According to HD for 24 Months of this Study}

The follow-up screening and diagnosis activities in Table 3 show the following:

- The Bibanga HD: the utilisation rate was higher than in the other two HDs; 4347 HAT RDTs were performed. Of these, only 136 (HAT RDT-positive rate 3\%) were positive. Ninety-three (or a proportion of $68 \%$ ) of these subjects reached a confirmatory facility. The screening rate was $3 \%$.

- Kongolo HD: the utilisation rate was the weakest compared with the other two HDs; 5496 patients received a HAT RDT. Of these, 162 (HAT RDT-positive rate 2.9\%) tested positive and 74 (or a proportion of $45.6 \%$ ) reached a confirmatory facility. In this HD, the screening rate was $11 \%$.

- Yasa Bonga HD: 6465 HAT RDTs were performed in fixed health facilities of Yasa Bonga. Only 146 subjects tested positive, i.e. the HAT RDT-positive rate was $2.2 \%$. Of these 146 seropositives, 115 (or a proportion of $76 \%$ ) underwent a parasitological confirmatory examination. In this HD, the utilisation rate was 0.18 for the 1 st year and 0.2 for the 2 nd year. The screening rate was $10 \%$.

It is worth mentioning that all parasitologically confirmed seropositives were treated.

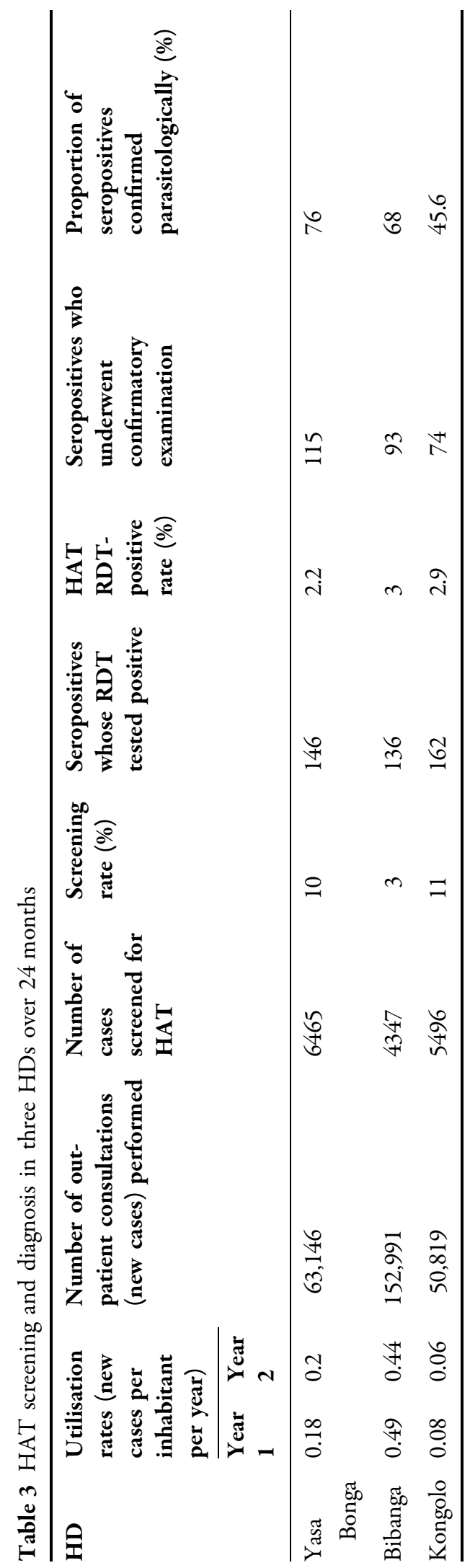


Influence of the Distance Between Screening and Diagnostic Confirmation Site on the Number of Confirmed Seropositives

Tables 4 and 5 present the results of the comparison of the distance between the screening and confirmation site for unconfirmed and confirmed seropositives and those who did not attend. The result reveals that for all HDs the variances in the considered samples were significantly different among them $(p<0.05)$ (Table 4 ). Therefore, the Student's test would take into account the inequality of variances.

Table 5 shows clearly that:

- in the Bibanga HD, the arrival of seropositives was largely associated with the short distance $(3.3 \mathrm{~km}$ vs. $10.6 \mathrm{~km})(p=0.000)$;
- in the Yasa Bonga HD, the arrival or not of seropositives did not depend on distance $(p=0.337)$;

- in Kongolo HD, the arrival of seropositives to the confirmation centre was significantly associated with the long distance $(55.5 \mathrm{~km}$ vs. $39 \mathrm{~km})(p=0.030)$.

\section{Status of RDT HAT Stock Management in the Three HDs During 24 Months of the Study}

Passive screening activities in the primary health centres of the three HDs experienced stock-outs for HAT RDTs (Fig. 4). Figure 4 shows the availability of HAT RDT for 24 months. The analysis shows that HAT RDT availability rates (RAPs) were $\geq 75 \%$ for all HDs: $79 \%$ in Yasa Bonga and $75 \%$ at Bibanga and Kongolo.

Table 4 Comparison of the distance between the screening centre and the confirmation site for seropositives that visited and were confirmed and non-confirmed seropositives in the three HDs using Levene's test for variance equality

\begin{tabular}{lllllll}
\hline Health district & Arrival & $\boldsymbol{N}$ (sample) & Average in $\mathbf{k m}$ & Variance & $\boldsymbol{F}$ test & $\boldsymbol{p}$ value \\
\hline Bibanga & No & 43 & 10.6 & 10.6 & 21.474 & 0.000 \\
& Yes & 93 & 3.3 & 6.6 & & \\
Kongolo & No & 88 & 55.4 & 51.6 & 5.888 & 0.016 \\
& Yes & 74 & 14.8 & 8.1 & & \\
\multirow{2}{*}{ Yasa Bonga } & No & 31 & 14.8 & 8.1 & 6.681 & 0.011 \\
& Yes & 115 & 13.0 & 12.5 & & \\
\hline
\end{tabular}

Table 5 Comparison of the distance between the screening centre and the confirmation site for seropositives that visited and were confirmed and non-confirmed seropositives in the three HDs using the $t$-test for equality of averages

\begin{tabular}{lllllll}
\hline Health district & Arrival & $\boldsymbol{N}$ (sample) & Average in $\mathbf{k m}$ & Variance & $\boldsymbol{T}$ test & $\boldsymbol{p}$ value \\
\hline Bibanga & No & 43 & 10.6 & 10.6 & 4.169 & 0.000 \\
& Yes & 93 & 3.3 & 6.6 & & \\
Kongolo & No & 88 & 39.2 & 40.2 & -2.191 & 0.030 \\
& Yes & 74 & 55.4 & 51.6 & & \\
Yasa Bonga & No & 31 & 14.8 & 8.1 & 0.760 & 0.337 \\
& Yes & 115 & 13.0 & 12.5 & & \\
\hline
\end{tabular}




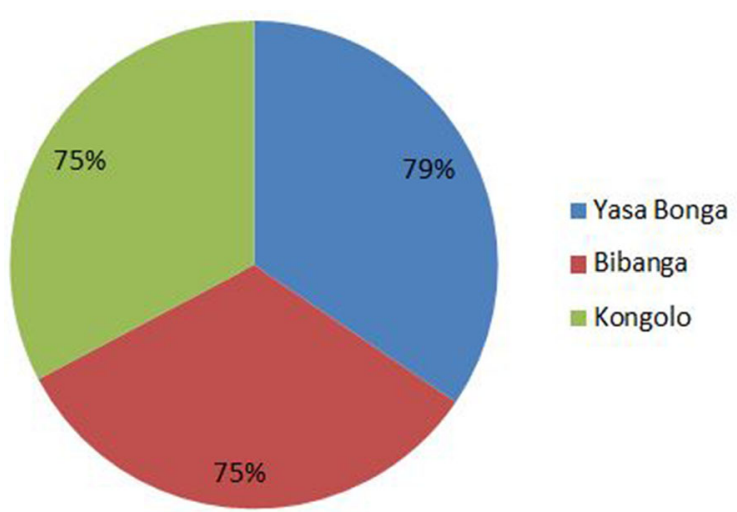

Fig. 4 Availability of RDT HAT in the three HDs during 24 months of the study

\section{Opinions of the Actors Involved in the Integrated HAT Activities in the HDs}

Five themes emerged from the collected data: the almost complete appropriation of the integration process of HAT activities by the HD, the community's weak awareness campaigns on integrated HAT activities, the quality of health services, obstacles to access to care and the socio-cultural point of view of the population. These elements influence each other and can negatively affect the integration of HAT activities as described below.

\section{Staff of the Health Districts Take Over Almost All Activities to Control HAT in the Area}

The health care providers (doctors and nurses) appreciated the screening activities in primary health centres because of the easy-to-handle tools, HAT RDTs, and an algorithm to follow for the screening and diagnosis of HAT. Nevertheless, many among them asked for an allowance as they were underpaid. For the HD supervising team, they declared that they had integrated the HAT control activities in their annual planning. They supervise HAT activities in primary health centres and report them during monthly assessment meetings. However, many of the interviewed team members said that their colleagues' performance was weak sometimes because they lacked motivation.

"...the management team bothers me; she believes that I am motivated and they are not motivated. And there is only me and some who are interested in the activities of the HAT". (INT YB supervisor nurse of HD)

This motivation request was because the majority of the health staff were underpaid and few thought the allowances in the health districts were sufficient.

\section{Weak Awareness Campaigns of the Community About Integrated HAT Activities}

The involvement of some community workers whose role was to increase public awareness was noticeable. They had a prior briefing on HAT by the treatment providers. However, some health professionals and community workers mentioned that the population was not informed because of a the community worker's lack of knowledge about HAT.

"HAT activities are integrated but some community workers were not involved and did not assist with providing awareness campaigns with correct information about this disease". (INT KG 2 head nurse)

In this survey, some members of the community declared that their primary health centres were not informed about the HAT screening activities and others asserted not even having received the information to go to the hospital or to a referral structure once the test was positive. They stated that often after having thought about malaria at home, because of headaches and fever, they could then attend a health facility. Only after the consultation did they discover to their astonishment that they had been declared seropositive for sleeping sickness.

“...all this time, I did not know; I came only to get care because I had a fever. They tested me and found that I had this disease. I was amazed. They wrote me a note and handed it to me and told me to go to a big hospital for another test". (INT KG 1 seropositive)

The community workers' lack of basic education certainly had consequences for community awareness. A basic training related to HAT for these health volunteers would be a motivation that would contribute to awareness. 


\section{Quality of Health Services}

The availability of HAT screening in endemic HDs was considered favourably by all health professionals. The health post, which is the most peripheral operation health unit, could recognise the clinical signs and refer the patients to the health centres for serological testing. However, some health professionals mentioned the absence of permanent parasitological confirmation services in some HDs. Although these services exist in some HD locations, some regulation of medical deontology needed to be enhanced for the trained health staff. Members of the management team in other HDs indicated that trained staff turnover also played a role in the quality of care.

"You know that there has been quite some staff turnover, that is something we do not plan". (INT KG doctor)

“...providers were late, you may arrive in the morning, and now on you are welcome even at 10 o'clock; that disheartens you to go there". (INT BI 2 seropositive)

Health professionals mentioned HAT RDT stock shortages. Those shortages hampered the use of the screening algorithm at primary health centres and even discouraged some health professionals. In addition, members of the supervising team of some HDs indicated the existence of weak collaboration with the experts in the programme (PNLTHA).

"... at the health centre and at the GRH, the great obstacle is the shortage in products, usually in RDT, once you have a shortage, but you have cases which fit the algorithm, but you do not have that possibility to realise the serological test". (INT BI doctor)

Problems related to the relevant resource supply for passive screening such as the HAT RDT and the weak supervision of integrated activities by HAT experts are some of elements that have an influence on quality of care.

\section{Obstacles to Access to Care}

Opinions of the community and health professionals about geographical and financial accessibility to the referral structures converged. These were the structures performing parasitological confirmation. Most of these structures were erratic. The seropositive patients needed to travel several kilometres to reach these structures. Some seropositives said they did not have the means to reach the referral facility and to ensure they had enough food. Even though HAT treatment is free of charge, the rest of the medical hospitalisation fees are not.

"...if we could not arrive up to now (to the referral centre $\mathrm{NB}$ ), it is just a lack of means. You see, we no longer have parents, we are living just like that. To arrive there, you should have money and this delays us to reach the general hospital". (INT KG 3 seropositive)

The great difficulty noticed during interviews was usually at the level of the parasitological confirmation facilities, which were perceived as geographically and financially unreachable for some seropositives.

\section{Socio-cultural Consideration of the Community}

Opinions of some survey participants clearly showed that some seropositives could easily attend health centres when they were suffering. They could accept any proposed therapy scheme. Survey participants also indicated the existence of a given category of persons who were informed about HAT screening via community workers or by the staff in charge of health care. Another category was that of those who were frustrated just because they were declared "seropositives". In this category, the seropositives were going to either traditional practitioners or churches. They persisted in believing that HAT is a pathology of supernatural origin. The latter, once declared "seropositive", might not return for the follow-up because they were afraid of the lumbar puncture or were ashamed as people could know that they had the disease.

"I didn't go to the hospital because of the back injection because it weakens the back and it will prevent me from working". (INT YB 3 seropositive) 
In short, socio-cultural beliefs about HAT persist. This evidence was clear as it was from opinions of community members and head nurses of health centres.

\section{DISCUSSION}

The results of this survey show that the primary health services of the three HDs performed passive HAT screening using the HAT RDT. The results of that screening were different from one HD to another. The utilisation rate of the services was very low in all HDs when we considered what was normatively expected for rural health care institutions in the Democratic Republic of the Congo. The expected utilisation rate in the DRC varies between 0.5 and 1 per person yearly for a new case. This average is ten times higher than what was found in Kongolo. This is an indication that the "coverage" of outpatient consultation is very poor and probably that many HAT patients simply do not use these facilities and thus remain undetected. Therefore, they are not detected. In the DRC, some HDs have support from external partners to complete certain activities. However, many health structures do not benefit from this support. Kongolo HD did not have shareholders to support its activities. This could be a reason for the reduced use of services compared with other HDs. The integration of activities in a system that does not function normally is not the main issue [29]. The weak use of curative services in the present context and the declining prevalence of HAT are weaknesses in the passive screening services in primary health centres. As suggested by other authors, the combination of passive and active screenings while taking into account the epidemiological context and the characteristics of the health system is a good option to assure the monitoring of HAT $[3,20,23]$.

Discrepancies in screening rates were remarkable in the three HDs. The Bibanga HD has a weak rate (3\%) though it has higher curative attendance than the other two. As some quotes about the qualitative research show, this situation may be due to the weak motivation of the health actors who are often underpaid and need extra income. This situation of health workers in HD may explain the lack of motivation about HAT activities.

The HAT RDT-positive rates were also different in the three HDs (2.2\% for Yasa Bonga, 2.9\% for Kongolo and 3\% for Bibanga). These rates were affected by the number of completed HAT RDTs in each HD, according to the algorithm; adherence to this algorithm depended largely on health care providers. Concerning the proportion of declared seropositives who underwent confirmation tests, the figures also differed from one HD to another (76\% for Yasa Bonga, $68 \%$ for Bibanga and $45.6 \%$ for Kongolo). This low percentage in Kongolo was due to the fact that this HD had only one referral facility (GRH) for all pathologies. The seropositives who could undergo parasitological tests were those with means (of transportation and to survive far from their residence) to reach the hospital. In all HDs, participants in the qualitative study also mentioned the lack of financial means to reach the referral structures. People affected by this pathology are very often poor and live in remote areas [30]. At this level, a possible intervention would be to go to these people for parasitological confirmation.

Distance analysis between the screening and confirmation structures indicated that in Bibanga HD the arrival of seropositives at the confirmation centre was significantly associated with the short distance $(3.3 \mathrm{~km}$ vs. $10.6 \mathrm{~km})$. This result is close to King's theory, which clearly asserts that the use of services lessens with distance [31]. The results of the qualitative survey show that seropositives were willing to comply with the directives they received at the health centres to undergo parasitological tests. In Kongolo $\mathrm{HD}$, the arrival of seropositives to the confirmation centre was significantly associated with the long distance $(55.5 \mathrm{~km}$ vs. $39 \mathrm{~km})$. Such a situation is understood in that in an HD where patients have to resort only to one referral facility for all illnesses, the arrival of seropositives depends upon at their own ability to continue with the cure. Moreover, the qualitative research shows that a lack of means for travel and subsistence during a possible hospitalisation was a major issue related to HAT. The long distance for reaching the confirmation centre was a major issue for some seropositives. 
In such an $\mathrm{HD}$, the implementation of extra referral institutions would reduce the distance seropositives need to travel. This was demonstrated by Wamboga et al. [9]. A collaboration between the 'PNLTHA' and stakeholders should be done before any implementation of HAT activities in health structures; certain requirements and good functionality of the health centres should be met, such as having relevant and reliable equipment, availability of qualified staff, low turnover of staff, costs, etc.). Implementing passive HAT screening activities in such an HD can be combined with reactive screening. In Yasa Bonga district, the arrival or not of seropositives did not depend on distance. Such a situation would be in relation to the seropositive behaviour. The qualitative research also indicates the existence of unfounded beliefs about HAT in this area (for instance, that a lumbar puncture would lead to an inability to work). In general, participants in this qualitative study showed a lack of HAT training for the community workers. This influences the awareness campaigns for the population. Seropositives who decide not attend the confirmation facility will lack information about the HAT diagnosis and care. Therefore, reinforcement of the awareness campaigns could contribute to increasing the use of confirmation structures. Different sources of health information should be combined especially in communities primarily using interpersonal and radio communication as their main source of information [32].

HAT RDT stock shortages impeded the integration process of passive screening in primary health services. The availability rate of HAT RDTs in Yasa Bonga was significantly different from those in the other HDs because of the closer location to Kinshasa. Participants in the qualitative survey also mentioned those shortages as an issue to be solved. These stock shortages could discourage health care providers and contribute to a loss of the community's trust in primary health care services.

Although all facilities had integrated the activities according to their technical abilities, only a few members of the HD management team were involved. The participants in our qualitative research sample indicated that the supervisions that were supposed to be integrated included a review of all activities during the site visits. The refusal to have this reported in this study may be a testimony to what Criel et al. [29] call "resistance to integration". The same reason is evident for HD management teams. Nevertheless, these teams are supposed to respond to their task of controlling endemic diseases [33].

To our knowledge, this is the first study to identify drivers of and obstacles to the integration process of HAT activities in primary health centres using qualitative research methods involving seropositive patients, community workers and health care staff. Although activities can be performed in primary health centres with well-adapted tools, the relatively low prevalence of the disease does not allow health staff in peripheral facilities to invest in keeping up good clinical skills for diagnosis and treatment of HAT [34].

\section{CONCLUSION}

Using HAT RDTs for passive screening in primary health care services is inevitably limited. Regarding the epidemiological context and obstacles to effective implementation of the integration process, it cannot by itself be an efficient alternative to the elimination of HAT as a public health problem by 2020 in DRC. The periodical mobile strategy, using the HDs' versatile staff trained in HAT, can be tested as it could work in synergy with integration. Therefore, significant resources should continue to be mobilised to achieve elimination of HAT by 2020, and even beyond. Studies should improve the present diagnostic tools and minimise travel costs for seropositives.

\section{ACKNOWLEDGEMENTS}

All participants in the study are thanked. We thank Jérémie Ilunga, Ibrahimu Brakit, Alain Funkisia, Julienne Tshowa, Jean-Pierre Kijana, Roger Kalo, Bonheur Tshiteku, Narcisse Tchowe and Nono Muba for their support in the data collection. We thank Paul Verlé and Raquel 
Inocencio Da Luz for their help in editing the manuscript. We also like to thank Bart Criel for the exchange on the concept of "integration".

Funding. We acknowledge the agency that provided financial support for this study, the Belgian Development Cooperation. The funder had no role in the study design, data collection and analysis, decision to publish or preparation of the manuscript. Philippe Mulenga received financial support thanks to a doctoral grant from the Belgian Development Cooperation under the FA4 agreement. Funding for the study and Rapid Service Fees was provided by the Epidemiology and Tropical Diseases Unit of the Institute of Tropical Medicine, Antwerp.

Authorship. All named authors meet the International Committee of Medical Journal Editors (ICMJE) criteria for authorship for this article, take responsibility for the integrity of the work as a whole and have given their approval for this version to be published.

Authors' Contributions. Philippe Mulenga, Pascal Lutumba, Oscar Luboya and Faustin Chenge drafted the study protocol. Philippe Mulenga conducted the data collection and analysed the data. Philippe Mulenga and Faustin Chenge wrote the first draft of the paper. All authors participated in its critical review. All authors endorse the final version and approve submission

Disclosures. Philippe Mulenga, Pascal Lutumba, Yves Coppieters, Alain Mpanya, Eric Mwamba-Miaka, Oscar Luboya and Faustin Chenge have nothing to declare.

Compliance with Ethics Guidelines. The study was approved by the Institutional Review Board Institute of Tropical Medicine Antwerp (no. 1156) and the Medical Ethics Committee of the University of Lubumbashi-DRC (UNILU/ CEM/2017/004). We received authorization from the Ministry of Public Health-DRC by the General Secretariat to carry out this study (MS no. 1251/SG/THA/2416/MK/2017) and local leaders also gave their oral consent. The ethics committee approved the verbal consent procedure, documented by audio recording. The information collected from the participants was treated confidentially and anonymised.

Data Availability. All authors had full access to all of the data in this study and take complete responsibility for the integrity of the data and accuracy of the data analysis. Qualitative and quantitative data can be made available upon request via Philippe Mulenga, drphilippe.mulenga@gmail.com.

Open Access. This article is distributed under the terms of the Creative Commons Attribution-NonCommercial 4.0 International License (http://creativecommons.org/licenses/ by-nc/4.0/), which permits any noncommercial use, distribution, and reproduction in any medium, provided you give appropriate credit to the original author(s) and the source, provide a link to the Creative Commons license, and indicate if changes were made.

\section{REFERENCES}

1. Simarro PP, Cecchi G, Franco J, et al. Estimating and mapping the population at risk of sleeping sickness. PLoS Negl Trop Dis. 2012;6(10):e1859.

2. Franco JR, Simarro PP, Diarra A, Jannin JG. Epidemiology of human African trypanosomiasis. Clin Epidemiol. 2014;6:257-75.

3. WHO. Control and surveillance of human African trypanosomiasis. Organ Tech Rep Ser. 2013;1-237.

4. Brun R, Blum J, Chappuis F, Burri C. Human African trypanosomiasis. Lancet. 2010;375(9709):148-59.

5. Franco J, Cecchi G, Priotto G, et al. Monitoring the elimination of human African trypanosomiasis: update to 2016. PLoS Negl Trop Dis. 2018;12(12):e0006890.

6. WHO. Accelerating work to overcome the global impact of neglected tropical diseases: a roadmap for implementation. Geneva: WHO; 2012.

7. Holmes P. First WHO meeting of stakeholders on elimination of gambiense Human African Trypanosomiasis. PLoS Negl Trop Dis. 2014;8(10):e3244.

8. The London Declaration on neglected tropical diseases. Uniting to Combat. 2012. https:// 
unitingtocombatntds.org/london-declarationneglected-tropical-diseases. Accessed Sept 25, 2018.

9. Wamboga C, Matovu E, Bessell P, et al. Enhanced passive screening and diagnosis for gambiense human African trypanosomiasis in north-western Uganda moving towards elimination. PLoS One. 2017;12(10):e0186429.

10. Blum J, Schmid C, Burri C. Clinical aspects of 2541 patients with second stage human African trypanosomiasis. Acta Trop. 2006;97:55-64.

11. Buguet A, Bourdon L, Bisser S, Chapotot F, Radomski M, Dumas M. Sleeping sickness: major disorders of circadian rhythm. Med Trop. 2001;61:328-39.

12. Kennedy PG. Human African trypanosomiasisneurological aspects. J Neurol. 2006;253(4):411-6.

13. Buscher P, Cecchi G, Jamonneau V, Priotto G. Human African trypanosomiasis. Lancet. 2017;390(10110):2397-409.

14. Boelaert M, Mukendi D, Bottieau E, et al. A phase III diagnostic accuracy study of a rapid diagnostic test for diagnosis of second-stage human African trypanosomiasis in the Democratic Republic of the Congo. EBioMedicine. 2018;27:11-7.

15. Checchi F, Chappuis F, Karunakara U, Priotto G, Chandramohan D. Accuracy of five algorithms to diagnose gambiense human African trypanosomiasis. PLoS Negl Trop Dis. 2011;5(7):e1233.

16. Buscher P, Gilleman Q, Lejon V. Rapid diagnostic test for sleeping sickness. $\mathrm{N}$ Engl $\mathrm{J}$ Med. 2013;368(11):1069-70.

17. Bisser S, Lumbala C, Nguertoum E, et al. Sensitivity and specificity of a prototype rapid diagnostic test for the detection of Trypanosoma brucei gambiense infection: a multi-centric prospective study. PLoS Negl Trop Dis. 2016;10(4):e0004608.

18. Kennedy PG. Clinical features, diagnosis, and treatment of human African trypanosomiasis (sleeping sickness). Lancet Neurol. 2013;12(2):186-94.

19. Mitashi P, Hasker E, Lejon V, et al. Human African trypanosomiasis diagnosis in first-line health services of endemic countries, a systematic review. PLoS Negl Trop Dis. 2012;6(11):e1919.

20. Simarro PP, Diarra A, Ruiz Postigo JA, Franco JR, Jannin JG. The human African trypanosomiasis control and surveillance programme of the World Health Organization 2000-2009: the way forward. PLoS Negl Trop Dis. 2011;5(2):e1007.

21. Mitashi P, Hasker E, Mbo F, et al. integration of diagnosis and treatment of sleeping sickness in primary healthcare facilities in the Democratic Republic of the Congo. Trop Med Int Health. 2015;20(1):98-105.

22. WHO. Control and surveillance of African trypanosomiasis. Organ Tech Rep Ser. 1998;881:1-114.

23. Simarro PP, Cecchi G, Franco JR, et al. Monitoring the progress towards the elimination of gambiense human African trypanosomiasis. PLoS Negl Trop Dis. 2015;9(6):e0003785.

24. Minisanté. Stratégie de Renforcement du Système de santé Edited by Minisanté. Kinshasa. 2006; 50.

25. Lumbala C, Simarro PP, Cecchi G, et al. Human African trypanosomiasis in the Democratic Republic of the Congo: disease distribution and risk. Int J Health Geogr. 2015;14:20.

26. PNLTHA. Déclaration de la politique de lutte contre la Trypanosomiase Humaine Africaine en République Démocratique du Congo. Volume $2^{\circ}$ Edition. Edited by PNLTHA. Kinshasa: PNLTHA; 2015.

27. Kohn L, Christiaens W. Les méthodes de recherches qualitatives dans la recherche en soins de santé: apports et croyances. Reflets et perspectives de la vie économique. 2014;53(4):67-82.

28. Tong A, Sainsbury P, Graig J. Consolidated criteria for reporting qualitative research (COREQ): a 32-item checklist for interviews and focus groups. Int J Qual Health Care. 2007;19:349-57.

29. Criel B, De Brouwere V. Conditions, limites et potentiel de l'intégration. In: Van Lerberghe W, Béthune X, editors. Intégration et Recherche, vol. 8. Antwerpen: Studies in Health Services Organisation \& Policy; 1998. p. 95-123.

30. Mumba D, Bohorquez E, Messina J, et al. Prevalence of human African trypanosomiasis in the Democratic Republic of the Congo. PLoS Negl Trop Dis. 2011;5(8):e1246.

31. Criel B. Organisation des Services de Santé Belgique: Institut de Médecine Tropicale d'anvers: Belgique. $2015 ; 49$.

32. Mpanya A. Facteurs socioculturels et contrôle de la Trypanosomiase Humaine Africaine en République Démocratique du Congo. Université Libre de Bruxelles, Belgique. Public health; 2015.

33. Minisanté. Recueil des normes de la zone de santé. Kinshasa, RDC. 2006.

34. Marchal B, Van Dormael M, Pirard M, Cavalli A, Kegels G, Polman K. Neglected tropical disease (NTD) control in health systems: the interface between programmes and general health services. Acta Trop. 2011;1:S177-85. 